\title{
DE LA AGROECOLOGÍA MAYA A LA ARQUEOLOGÍA DEMOGRÁFICA: ¿CUÁNTAS CASAS POR FAMILIA?
}

\author{
María de Guadalupe Zetina GutiérRez \\ Doctorado en Antropología \\ Instituto de Investigaciones Antropológicas \\ Universidad Nacional Autónoma de México
}

\author{
Betty Bernice Faust \\ Investigadora independiente
}

\begin{abstract}
Resumen: Durante 2006, en Pich, Campeche, se recuperaron 31 historias orales enfocadas en los patrones de movilidad entre familias agricultoras que viven en rancherías establecidas cerca de fuentes permanentes de agua. Se identificaron dos patrones: 1) el movimiento de una ranchería a otra cada 14 años, en promedio, con reocupación en un ciclo generacional; y 2) movimientos repetidos durante el año entre la casa en la ranchería y otra en el pueblo (bilocalidad). Así, en un ciclo doméstico de aproximadamente 35 años, la típica familia agricultora ocupaba por lo menos tres casas. Esto sugiere que las estimaciones del tamaño de las poblaciones agrícolas que suministraban alimentos a las ciudades-estado mayas en las Tierras Bajas del Norte deberían ser revisadas y ajustadas. Hasta ahora dichos cálculos no han incluido una tasa de reducción basada en información etnográfica detallada como la que presentamos ahora sobre las familias agricultoras de Pich.
\end{abstract}

Palabras clave: mayas yucatecos, agroecología, arqueología demográfica, agricultura nómada, familias milperas.

ABSTRACT: During 2006, thirty-one oral histories were collected in Pich, Campeche, to research patterns of mobility among agricultural families living in hamlets (rancherías) located near permanent water sources. Two patterns were found: (1) movement from one hamlet to another on average every fourteen years with reoccupation in a generational cycle; and (2) repeated movements throughout the year between the hamlet home and a permanent house in town (dual-residence). Thus, over a domestic cycle of approximately thirty-five years, the typical agricultural family occupied at least three houses. These data suggest a need to revise existing Pre-Columbian population estimates for the agricultural population providing food to the citystates of the Northern Maya Lowlands. In general, estimates based on house mounds have not included rates of reduction due to mobility or dual residency for lack of detailed ethnographic information such as we here provide for the agricultural families of Pich.

KEYwords: Maya Yucatecan, Agro-ecology, Demographic archeology, Nomadic agriculture, Milpa families.

ReCEPCIÓN: 17 de septiembre del 2008.

ACEPTACIÓN: 18 de junio del 2009. 



\title{
DE LA AGROECOLOGÍA MAYA A LA ARQUEOLOGÍA DEMOGRÁFICA: ¿CUÁNTAS CASAS POR FAMILIA?"
}

\author{
María de Guadalupe Zetina GutiérRez \\ Doctorado en Antropología \\ Instituto de Investigaciones Antropológicas \\ Universidad Nacional Autónoma de México \\ Betty Bernice Faust \\ Investigadora independiente
}

\section{Introducción}

En Pich, una población de herencia cultural mayormente maya que se mantuvo en relativo aislamiento demográfico y tecnológico hasta la década de 1960, $75 \%$ de los entrevistados nacidos entre 1906 y 1936 parece haber participado en patrones de movimiento ${ }^{1}$ y bilocalidad ${ }^{2}$ relacionados con la agricultura maya "tradicional", lo cual concuerda con lo reportado en otras partes de las Tierras Bajas Mayas (Bracamonte y Sosa, 2001; Brown, 1993; Farriss, 1984; Faust, 1998; Redfield y Villa Rojas, 1971 [1934]; Norman Schwartz, comunicación personal, 2006; Tax, 1937).

Por ello, argumentamos que las cifras paleodemográficas que hasta hoy han estimado los arqueólogos en la península de Yucatán usando la técnica de conteo

\footnotetext{
Este artículo está basado en los resultados obtenidos en la tesis de maestría en ciencias, especialidad en ecología humana, de la arqueóloga Guadalupe Zetina bajo la dirección de la etnoecóloga doctora Betty Faust en el Centro de Investigación de Estudios Avanzados del Instituto Politécnico Nacional (Cinvestav-IPN), Mérida, Yucatán. El desarrollo de la investigación estuvo asesorado por el edafólogo doctor Heriberto Cuanalo, el hidrobiólogo doctor Eduardo Batllori del Departamento de Ecología Humana (Cinvestav) y los arqueólogos doctor William Folan y doctor Armando Anaya del Centro de Investigaciones Históricas y Sociales (Cirs) de la Universidad Autónoma de Campeche (Uacam).

${ }^{1}$ Este patrón tradicional fue modificado entre 1960 y 1980 por la introducción de uno comercial por empresarios locales (patrones) que basaban sus ganancias en la venta de maíz y maderas preciosas y semipreciosas en Campeche, explotando grandes territorios con milpas y con el corte de madera. El uso de los terrenos agrícolas, en este caso, fue lineal y no cíclico ni sustentable; el movimiento de personas ocurrió hacia el sur para cortar los montes altos y usar los suelos que habían recuperado su fertilidad durante mucho tiempo sin uso humano (posiblemente desde el siglo x).

${ }^{2}$ Si la familia vive en el pueblo el milpero va y se queda por solamente estas temporadas a pernoctar cerca de su milpa. Si toda la familia vive en una ranchería entonces es la familia la que regresa al pueblo para ir a fiestas, realizar visitas familiares y acudir al mercado; aunque a veces regresan por enfermedad u otras razones personales.
} 
de unidades residenciales ${ }^{3}$ en contextos regionales ${ }^{4}$ deberían reducirse entre dos y tres, que es el número mínimo de casas que una familia campesina construye y abandona en un lapso aproximado de 35 años. Por supuesto que reconocemos que habría que ajustar este factor según la evidencia acerca del transporte de alimentos a distancia, el porcentaje aproximado de la población especialista no productora de alimentos y la intensificación agrícola documentada en el área (ver la discusión teórica). Sin embargo, en las regiones sin evidencia de intensificación agrícola la agricultura de roza, tumba y quema parece haber sido la única usada.

En este trabajo nos enfocaremos en la movilidad y bilocalidad relacionadas con la agricultura maya documentada etnográficamente en Pich, Campeche, para entender los patrones arqueológicos, materiales y espaciales, que son la base para las estimaciones del tamaño de paleopoblaciones.

\section{Discusión teórica: población y agricultura maya}

Durante la primera mitad del siglo xx fue común pensar que la agricultura tradicional de los mayas yucatecos sólo permitía comunidades dispersas de baja densidad y centros ceremoniales ocupados cíclicamente (Harris, 1972). Estas ideas se basaron en las descripciones del cronista fray Diego de Landa (1997, original 1566) en las que cada agricultor ocupaba sus milpas durante dos años seguidos con un periodo de descanso largo. En publicaciones posteriores como la de Redfield y Villa Rojas (1962, original 1934), Pérez Toro (1942) y otras de agrónomos y etnógrafos de la época se apoyó esta visión de una población dispersa y baja.

No obstante, el descubrimiento de numerosos cimientos de antiguas casas mayas en los alrededores de lo que entonces se creía eran los centros ceremoniales a partir de la década de 1930 (Culbert y Rice, 1990: 9-10) permitió a William Haviland (1969: 40) estimar que Tikal durante el periodo Clásico tuvo una densidad de población de entre 600 y 700 pers. $/ \mathrm{km}^{2}$ en el área nuclear y 300 pers. $/ \mathrm{km}^{2}$ en el resto de la ciudad. Por su parte, William Sanders (1968) estimó 400 pers./ $\mathrm{km}^{2}$ en la misma ciudad y 200 pers. $/ \mathrm{km}^{2}$ en la periferia. Kintz y Fletcher (1983: 196-210) estimaron la densidad de población de Cobá durante el Clásico Tardío en 635 pers. $/ \mathrm{km}^{2}$. En el libro que editaron sobre el tema, Culbert y Rice (1990) presentaron su interpretación de los datos hasta entonces existentes, según la

\footnotetext{
${ }^{3}$ La técnica de conteo de unidades residenciales consiste en multiplicar el número de casas que se piensa fueron contemporáneas por una figura del tamaño de la familia, generalmente entre 4 (recomendado por Sanders y Price, 1968) y 5.6 (con base en los datos de Redfield y Villa Rojas, 1962 [original 1934]) para la península de Yucatán. Para otras regiones se han usado otros tamaños de familia, de acuerdo con los datos etnohistóricos y etnográficos disponibles. Posteriormente se hacen ajustes de acuerdo a las estructuras ocultas, abandonadas o reusadas, etcétera.

${ }^{4}$ Se entienden como "contextos regionales" áreas o territorios con comunidades o asentamientos con una variedad de rangos (ciudades, pueblos y rancherías).
} 
cual los mayas tuvieron una de las poblaciones más densas del mundo preindustrial, como las de Java o China. Esto es difícil de creer debido a que en estos países asiáticos se contaba con animales de tiro, arado y carreta, mientras que los mayas dependían solamente de la mano de obra humana para el transporte y la producción de alimentos.

Es obvio también que esta densidad no podría haberse mantenido por el sistema agrícola maya de roza, tumba y quema. Harrison (1977: 479) reportó que a nivel mundial 77 pers. $/ \mathrm{km}^{2}$ es el máximo sostenimiento de la agricultura de roza, tumba y quema en condiciones óptimas de suelos y clima. Más tarde Faust y Bilsborrow (2000), a través de una revisión de los datos de cuatro reportes etnográficos de la península de Yucatán, concluyeron que en esta región (con suelos de poca profundidad, un subsuelo kárstico y seis meses al año de sequía), la máxima población que puede mantener la roza, tumba y quema es de 25 personas por km² (cf. Gutiérrez Martínez, 1993: 36; Gómez-Pompa et al., 2003: 4).

Así que es obvio que para sostener las grandes poblaciones fueron necesarios los sistemas agrícolas intensivos cuya evidencia los investigadores buscaron y encontraron a partir de la década de 1970. Estos restos de sistemas agrícolas intensivos incluyeron terrazas (Turner, 1979, 1983), canales asociados con campos elevados o drenados en bajos (Harrison, 1977, 1993; Scarborough, 1983) y otros asociados con la desviación y el aprovechamiento de los cauces de los ríos (Siemens y Puleston, 1972).

Estos fueron los primeros indicios del surgimiento de un nuevo paradigma acerca de la agricultura maya que se consolidó con la publicación en 1978 del libro Pre-Hispanic Maya Agriculture, editado por Harrison y Turner, seguido en 1982 por Maya Subsistence, editado por Kent Flannery. En 1983, Ray Matheny et al. reportaron un sistema complejo y grande de canales y depósitos de agua en Edzná construido durante el Preclásico (a $20 \mathrm{~km}$ de nuestra área de estudio), pero no tuvieron tiempo para buscar las huellas de campos elevados, aunque indicaron que la cantidad de agua manejada fue mucho mayor que la necesaria para el uso de la población. Al igual que Matheny et al. en Edzná, Mary Pohl (1990) encontró evidencia en Belice del uso de humedales y sus modificaciones con canales de drenaje desde el periodo Preclásico. En 1996, Fedick edita un libro con más de 20 capítulos sobre las varias modificaciones y usos de diferentes tipos de suelos, incluyendo las terrazas y los campos drenados por canales además de otros tipos de modificaciones prehispánicas no reportadas anteriormente. En 2003, GómezPompa et al. editan un libro con más información sobre estos sistemas de intensificación agrícola y el manejo de la selva, humedales y otros recursos naturales por los mayas prehispánicos. En 2004, Kunen documenta varias modificaciones agrícolas de terrenos en el "Far West Bajo" de Belice, construidas durante el Preclásico y en uso hasta el Posclásico.

Pero a pesar de lo numerosa que parece ser la evidencia de intensificación agrícola y de su innegable importancia para abastecer a las grandes ciudades, sugerimos que la roza, tumba y quema continuó contribuyendo, en gran parte, al 
sostenimiento de la población durante los tres milenios de la civilización maya. Es importante resaltar que no en todas las áreas con alta densidad constructiva arqueológica se ha hallado evidencia de intensificación agrícola o ésta ha sido muy limitada, pues no corresponde con las altas cifras de población estimadas, por ejemplo, en la región Puuc (Becquelin y Michelet, 1994; Dunning, 1992; Tourtellot, Sabloff y Smyth, 1990).

La proporción de alimentos producida por la milpa de roza, tumba y quema dentro de la dieta debió ser mayor durante algunos periodos y en áreas de menos densidad poblacional pues requirió menos trabajo para producir la misma cantidad de alimentos que en los sistemas intensivos antiguos, si bien es claro que estos últimos la producen en menos terreno (Culbert, 1977: 518).

Es tema de otra investigación buscar evidencia que indique la proporción del alimento aportado por cada sistema agrícola y su relación con el incremento y decremento de la densidad poblacional. Ahora sólo podemos concluir que en temporadas de baja densidad de población no había razón para invertir tiempo y energía en construir y mantener terrazas y campos drenados. Así se documentó durante los primeros siglos de la Colonia cuando las epidemias, la violencia y los cambios ecológicos (debidos a la introducción de especies exóticas), disminuyeron notablemente a la población.

Una cuestión notable es que Robert Redfield y Alfonso Villa Rojas (1962: 5253) documentaron que el número de días ocupados en tareas de la milpa es alrededor de 72. Esto implica que los milperos mayas siempre podían tener otras ocupaciones en su pueblo, además de ir por temporadas a trabajar en sus centros rectores en labores comunales, como la construcción de obras públicas (Abrams, 1994).

Así que hay dos factores importantes a considerar en las estimaciones arqueológicas de densidad de población en cualquier periodo: 1) las proporciones de alimentos producidas por cada sistema y 2) el número de casas ocupadas por cada familia en su ciclo doméstico. Para lo segundo es importante notar la relación estrecha entre la agricultura de roza, tumba y quema, y la bilocalidad y el movimiento cíclico de familias entre rancherías. Esta relación debió haber existido también en tiempos prehispánicos como producto de un patrón de adaptación a este tipo de medio ambiente. No obstante, sin animales de carga y otros medios de transporte, probablemente las distancias entre rancherías, pueblos y centros urbanos fueron menores que las registradas en el mundo moderno, pero estos movimientos respondieron a las mismas necesidades de la agricultura de roza, tumba y quema aunque otros factores (sociales, económicos, religiosos, personales, etc.) también pudieron incidir.

Etnógrafos y etnohistoriadores han reportado esta preferencia cultural de mantener una segunda residencia en el pueblo o, en su caso, en la periferia de la ciudad con la intención de obtener alimentos. Durante tiempos prehispánicos este mismo principio parece haber funcionado (Farriss 1984: 319; Zetina, 2007: 119-122; 141). 


\section{Descripción del área de estudio}

Los datos fueron tomados de la comunidad maya de Pich, Campeche, México. Ésta se encuentra en el centro oeste de la península de Yucatán, a $85 \mathrm{~km}$ al sureste de la ciudad costera de Campeche y $20 \mathrm{~km}$ al sur de la metrópoli prehispánica de Edzná. Dicha comunidad se encuentra en el borde oriental del valle de Edzná a una altitud de 100 msnm (Faust, 1998; figura 1).

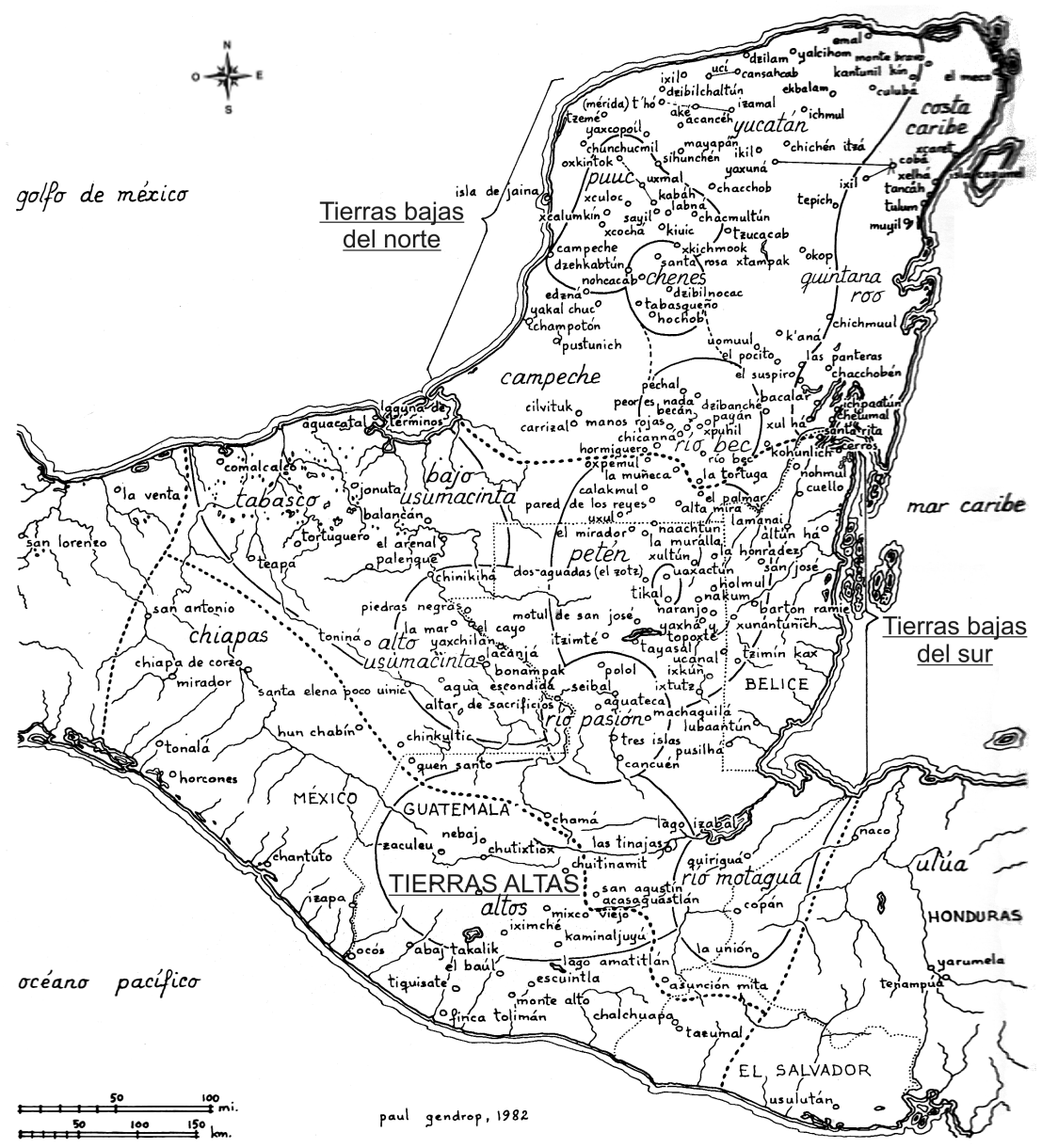

Figura 1. Ubicación del área de estudio en las Tierras Bajas del Norte.

Modificado de Gendrop, 1997: 25 
Después de más de una década de estudiar los procesos de cambio cultural dentro de esta comunidad maya, Faust reportó una continuidad importante de la cultura y la tecnología agrícola mayas desde tiempos prehispánicos hasta modernos. Argumentó que debido a su cercanía con Edzná y sus características materiales, se puede entender a Pich como parte de la esfera de influencia de esta capital regional y posiblemente como contribuyente a su alimentación en tiempos precolombinos (Faust, 1998: 27).

A través del desarrollo de las épocas colonial y moderna, la comunidad permaneció relativamente aislada ${ }^{5}$ hasta la construcción de los primeros tramos carreteros entre Campeche y Pich, a inicios de la década de 1960. Esta fue una razón importante para la conservación de costumbres y conocimientos mayas a pesar de que la mayoría de la población no se autoadscriba hoy en día como "maya" ni tampoco se hable esta lengua públicamente (Faust, 1998: 27). En la actualidad, la comunidad tiene una población que oscila alrededor de 2000 habitantes, descendientes principalmente de los grupos étnicos mayas, pero mezclados con minorías de españoles, africanos y chinos que llegaron para construir el ferrocarril (Faust, 1998; 2004).

\section{Antecedentes de la investigación y la metodología}

La movilidad y bilocalidad de los mayas de las Tierras Bajas han sido reportadas por etnohistoriadores y etnógrafos como un comportamiento relativamente común, como ya se ha mencionado. Destaca lo que apunta Farriss (1984: 318, 319) cuando menciona que sociedades agrarias premodernas como la maya parecen haber tenido mayor movilidad geográfica de lo que se había pensado; también es posible la existencia de una tendencia cultural hacia la dispersión que no se originó cuando se trató de huir del yugo colonial sino fue anterior (cf. Bracamonte y Sosa, 2001 sobre movilidad de rancherías durante la Colonia). Farriss (1984: 319) también reporta movilidad relacionada con bilocalidad entre pueblo y ranchería. Los reportes etnográficos más antiguos sobre bilocalidad vinculada con la agricultura de roza, tumba y quema datan de la década de los treinta (Redfield y Villa Rojas, 1962; Tax, 1937) en Yucatán y Guatemala. Le siguieron Brown (1993) en Yucatán otra vez y Faust $(1988 ; 1998)$ en Campeche.

No obstante, en la mayoría de las estimaciones paleodemográficas estos fenómenos han sido prácticamente ignorados, mientras una minoría considera la posibilidad de su existencia pero sin aplicar una tasa de reducción pues carecía de datos

\footnotetext{
${ }^{5}$ Los franciscanos establecieron la Misión de la Montaña en esta región a principios del siglo xVII. Durante tiempos coloniales varias haciendas y fincas fueron establecidas para la cría de ganado y la producción de caña de azúcar. Llegaron también patrones de la ciudad de Campeche que organizaron la extracción de palo de tinte, primero, y después del chicle. Así que Pich se encontraba incorporado a la economía mundial, pero sus habitantes también mantenían una economía local basada en la milpa de roza, tumba y quema, y en el uso de recursos naturales locales.
} 
concretos para hacerlo (Ford, 1990; Folan, Kintz y Fletcher, 1983; Culbert, 1990; Santley,1990; Tourtellot, 1990; Webster y Freter, 1990; Webster, Freter y Gonlin, 2000).

En 1985, durante su trabajo de campo, Faust descubrió bilocalidad estacional (una casa en el pueblo además de las de las rancherías) y un uso cíclico generacional de los terrenos agrícolas (Faust, 1988; 1998). Más tarde, esta etnógrafa junto con dos arqueólogos y un demógrafo (Folan et al., 2000: 9,10) propusieron que los restos de las rancherías prehispánicas podrían haber sido contabilizados erróneamente como unidades residenciales permanentes, provocando cifras demasiadas elevadas en los cálculos paleodemográficos mayas. La misma propuesta fue presentada otra vez por Faust en 2001, pero sin mucho eco hasta que durante el 2005 y el 2006, cuando Zetina —quien ya había realizado estimaciones de paleopoblaciones en la región Puuc (Zetina, 2003) y análisis a las técnicas de estimación del tamaño de las poblaciones prehispánicas (Zetina, 2003; 2004; y Faust 2006)—, retomó y profundizó en este tema (Zetina, 2007). La metodología consistió en una combinación de métodos de investigación social (observación participante, entrevistas semiabiertas o guiadas, diagramas de parentesco y análisis de documentos) y otras técnicas de registro espacial usadas en la arqueología y la geografía (Zetina, 2007: 67-70).

\section{La milpa tradicional y los patrones de movilidad y bilocalidad residencial en Pich, Campeche}

Antes de que existieran estudios etnográficos centrados en entender el mecanismo de la milpa maya tradicional, se presuponía que los agricultores no regresaban nunca al mismo terreno y que existía una destrucción "bárbara" continua e inevitable de la selva virgen. Ahora sabemos que muchas culturas han vivido por miles de años en un territorio conocido, usando un patrón cíclico similar al de los mayas y regresando a los terrenos usados anteriormente por sus abuelos o sus padres (Conklin, 1957; Harris, 1972; Netting, 1994: 329-340; Pool, 1997: 6-7).

El descanso de veinte años de la selva restaura las condiciones óptimas para la siembra a través del crecimiento de los retoños de los tocones y de las raíces así como de las semillas provenientes de la franja forestal. La sombra de la vegetación en recuperación elimina el zacate y las hierbas que compiten con los cultivos mientras que las raíces profundas de los árboles extraen minerales del subsuelo, dejándolos sobre el suelo en las hojas caídas. Las cenizas de su biomasa también contribuyen a la fertilidad. Sugerimos que fue por medio de estos servicios gratuitos de la sucesión secundaria como Culbert (1977: 518) encontró que la agricultura de roza, tumba y quema requiere menos trabajo para producir la misma cantidad de alimentos en comparación con los sistemas intensivos. ${ }^{6}$

\footnotetext{
${ }^{6}$ No hay que perder de vista que la ventaja de los sistemas intensivos es la de producir más cantidad en menos área, aunque con mayor inversión de trabajo por cantidad cosechada.
} 
La milpa considerada tradicional es aquella basada en un patrón cíclico de uso de los suelos agrícolas asociado con rancherías familiares y con la producción para autoconsumo (figura 2). En contraposición, está la milpa comercial con un patrón lineal de uso de terrenos agrícolas no sustentable y desarrollada junto con el auge del corte de madera a partir de 1960 (figura 3) (Zetina, 2007: 78-79).

En el patrón cíclico las milpas se mueven en un radio de $2 \mathrm{~km}$ tomando como centro una fuente de agua permanente (por lo general, aguadas ${ }^{7}$ medianas y grandes que puedan retener este líquido todo el año y, en menor medida, pozos). Sin embargo, en Pich, Campeche, el promedio de ocupación de las rancherías fue de catorce años, antes de trasladarse a otra aguada y construir una nueva ranchería (Zetina, 2007: 78-79, 138).

Es altamente significativo que de 371 individuos (reportados por los 31 informantes en diagramas de parentesco), de varios estratos económicos y seis diferentes generaciones, sólo el $25 \%$ se reportó sin movilidad ni bilocalidad ya que vivieron permanentemente en el pueblo (en una sola casa sin ningún tipo de movilidad). Con base en lo anterior, antes de 1960 Pich pudo tener aproximada-

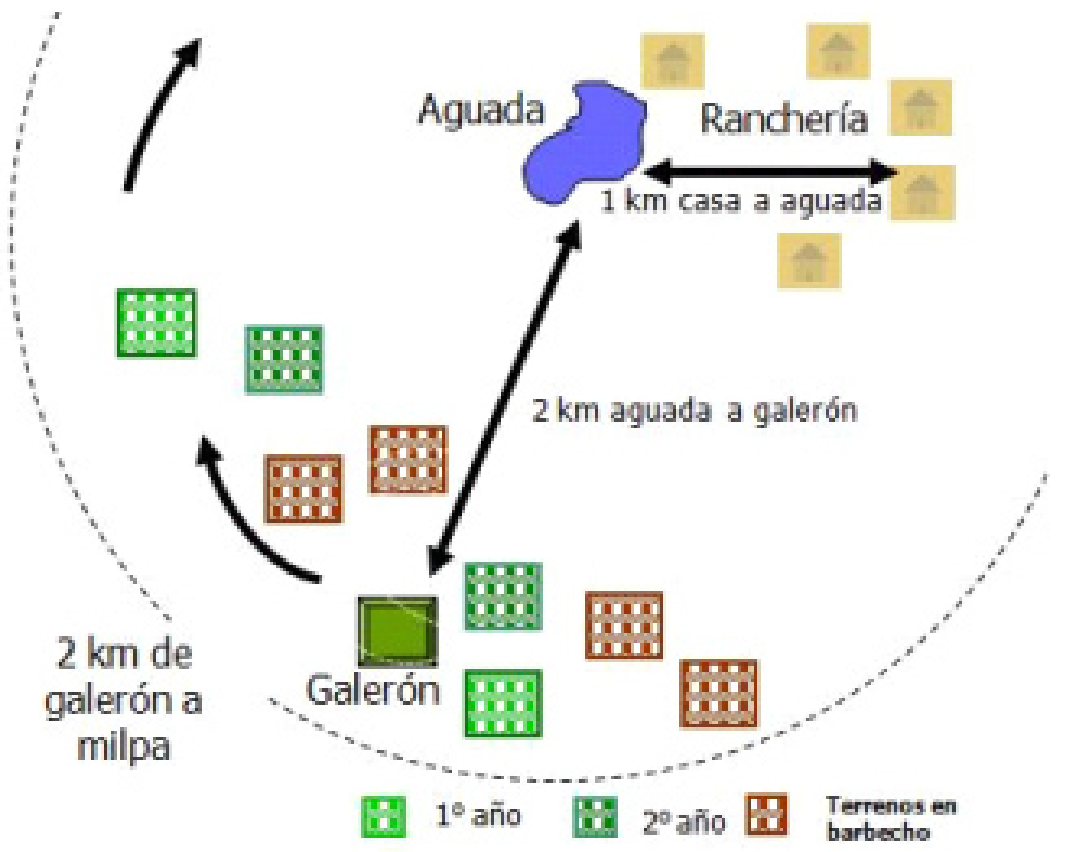

Figura 2. Esquema del patrón de movimiento circular de las milpas y del galerón milpero y de las rancherías asociados a una producción agrícola de autoconsumo (Zetina, 2007: 76)

\footnotetext{
${ }^{7}$ Depresiones formadas en los suelos calizos (karst) al pie de laderas, donde se almacena el agua de lluvia (Siemens, 1978: 136).
} 


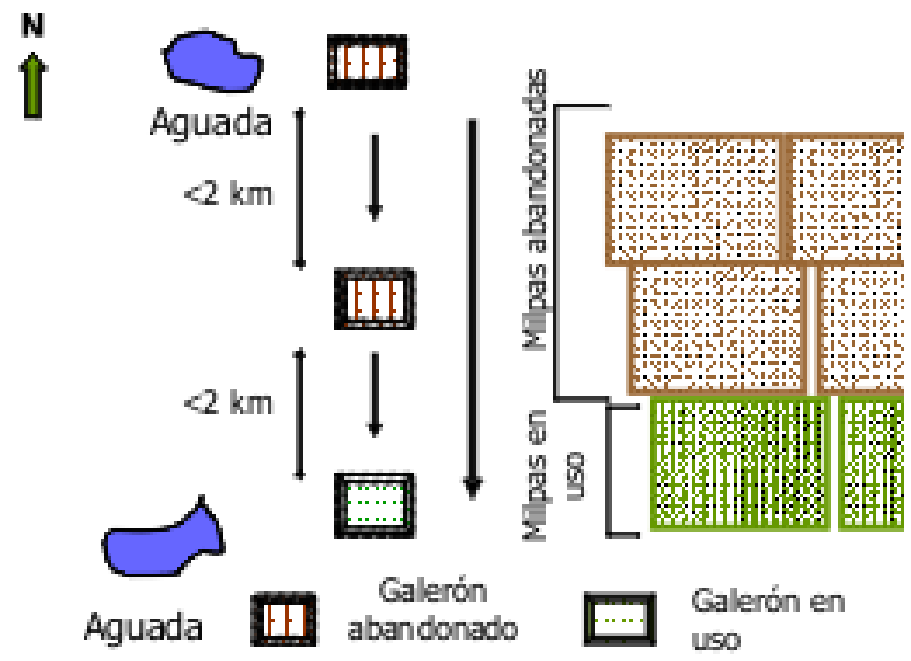

Figura 3. Esquema del patrón de movimiento lineal de las milpas y del galerón milpero asociado a una producción agrícola comercial (Zetina, 2007: 77)

mente una población móvil del $75 \%$ que aprovechaba los terrenos agrícolas en ciclos regulares transmitidos generacionalmente. Un caso muy claro de bilocalidad fue narrado por un informante de 53 años de edad (Zetina, 2007: 117):

El señor recién casado se fue a vivir a allá (en la ranchería con toda su familia) y estuvo como 15 años. Ya grandes sus hijos, bajó acá y los entregó a la escuela y aprendieron sus hijos. Ya tiene como unos 70 años el señor ese. Yo recuerdo que él vivió muchos años en esa aguada. Ese señor tenía gallinas, cochinos, abeja tenía allá. iTiene como 24 kilómetros! Tiene de Pich a esa aguada (pausa). Sí. Y tenía casa acá, también (en Pich).

Comparando la movilidad por generaciones, las dos más antiguas (nacidos entre 1916 y 1936) mantuvieron un porcentaje alto (75\%), mientras que las cuatro generaciones más recientes mostraron una reducción gradual debido a la introducción de los servicios básicos modernos (electrificación, escuelas, clínicas, agua potable, carreteras y la introducción de la milpa mecanizada con el uso del maíz "híbrido"), que fue anclándolas cada vez más en el "confort" de la modernidad (Zetina, 2007: 80). Al respecto, una mujer de 72 años de edad relató (Zetina, 2007: 117):

Bajamos acá a vivir (las mujeres de la familia) por el estudio de ellas (las hijas). Porque si estamos en el monte ya no pueden estudiar. iAja! En cambio le digo a él (al esposo): "Pues ni modo, hay que bajar en Pich a vivir por el estudio de las chiquitas", que ya estaban grandecitas. "Necesitan un poco de estudio" -le 
digo- "Porque si estamos aquí en el monte ¿a dónde van a estudiar? ¿Dónde van a estudiar?" Y después bajamos aquí a entregarlos a la escuela. Y le digo: "Ya no me puedo quedar en el monte" ¿quién los va a atender? iAja! (pausa) y me bajé (a Pich). Me bajé a vivir también acá y bajaron mis gallinas. Pero, ya no es igual. En el monte viven más bien los animales.

Una de las mayores ventajas de moverse regularmente fue la abundancia de animales para presas de caza (venado, jabalí y pavo de monte eran los favoritos) así como una mayor productividad agrícola asegurada por la presencia de árboles grandes que no habían sido cortados por décadas (Zetina, 2007: 84, 97); este fenómeno es muy similar a lo reportado por Meggers y Miller (2006) en la Amazonia. En regiones donde había menos competencia por el espacio debido a baja densidad de población las milpas podían tener un barbecho mayor a veinte años y, en teoría, no habría necesidad de mover los ranchos familiares. Sin embargo, la fauna no se recupera tan rápido y la milpa siempre fue más productiva en terrenos con barbechos de más de veinte años. Como menciona un pichuleño de 48 años de edad (Zetina, 2007: 73):

Sí, porque acá [en el pueblo de Pich] no se puede hacer milpa. Así [allá] tenías que hacer [la milpa] porque está cerca el monte. Pero [acá] casi no se producía como se produce allá.

Otro de 52 años dijo (Zetina, 2007: 83):

En un mecate cuando se producía daba [en la ranchería en el despoblado] dos, tres costales de mazorca. Daba un promedio de 150 kilos. Se sembraba frijol, se sembraba calabaza, camote, macal, ibes blancos. Todo se producía muy bien sin fertilizantes. Se sembraba tomate, sandía... Era nada más para comer, para traer acá [al pueblo de Pich, no a vender en la ciudad de Campeche].

Pero también existieron otras razones (Zetina, 2007: 97-99): 1) mayor calidad de vida, lo que significa menos enfermedades y mortalidad al evitar epidemias ${ }^{8}$ y una dieta amplia y variada, producto de la caza, los cultivos de la milpa y la recolección. Una mujer de 48 años mencionó:

Ahí se hacían los animales: gallinas, cochinos, pavos (pausa). Es bonito vivir en el monte porque no tienes que comprar nada de carne. Todo se producía porque

\footnotetext{
${ }^{8}$ Dos informantes reportaron que en la historia de Pich hubo epidemias de viruela, sarampión, tos ferina, cólera y tifoidea (las más mortales fueron las primeras dos). Otros dos informantes mencionaron que algunas rancherías aisladas surgieron cuando la gente huía de las epidemias y que algunos pueblos pequeños como Chuum y Yacachí desaparecieron por causa de éstas mismas (Zetina, 2007: 71). Faust (1988: 337-338) encontró documentos en el registro civil de Pich, Campeche, en 1986, que hablan sobre una epidemia de viruela negra en 1917 y 1918 que mató a 199 personas. Otro pueblo similar en tamaño a Pich, San Juan Cantemó, fue abandonado por esta epidemia según datos de campo de Faust recuperados en 2007.
} 
salen ellos [los hombres] y no tarda que se alejen así de ir y vienen con pizote, pavo, chachalaca, puerco de monte (pausa) iasí traen carne! Hay veces que traían armadillos, tepezcuintle (pausa), esos animales pa' comer. No se compraba nada. Solamente tenías que venir [a Pich], bajarte de allá y comprar mercancía para que puedas llevar allá, para que pueda comer la gente. Se compraba namás fab, jabón, manteca lo demás todo allá. iAhí crecen más saludables los niños! iNo se enferman! iNadie se enferma! iCuando bajen tus hijos hasta gordos están! Está más sano ahí. (pausa) iPuro aire puro estas respirando! No como aquí que hay muchas enfermedades a veces que padecen los niños.

2) Había menos conflictos con los vecinos al haber menos competencia por recursos y espacio. Una informante de 76 años de edad mencionó al respecto:

Yo no sé porque se fue allá lejos [mi esposo] porque antes había montes [cerca]. Sí, trabajaba aquí cerca también, pero como muchos tienen cochinos (y) no los llevan, entonces se meten a las milpas. (pausa) Destrozan las milpas, comen mucho maíz. Por eso mejor se fue más lejos a Chunzón, Chuncoloc. ${ }^{9}$

3) Dependencia mínima del sistema monetario ya que "no hacía falta comprar casi nada”. Un hombre de 54 años de edad mencionó:

Habían gente que no utilizaba ni lo esencial como papel, no tomaban café sino la tortilla, esa que queman y se ahorraban el café; el azúcar, no compraban azúcar tomaban miel. El maíz pues ahí lo tienen y hacían la tortilla. Carne, sí, había mucho, porque tiraban venado, pavo, faisán, puerco de monte. No... compraban nada. Entonces en ese tiempo la gente si juntaba sus centavitos porque no gastaba.

4) Motivos personales. El simple gusto de vivir en el monte con la naturaleza, conociendo lugares o al estar atravesando una situación económica difícil, esperando recuperar la solvencia económica para regresar al pueblo. Una mujer de 48 años dijo:

Se alegra uno porque está el monte. Allá en el monte no es como aquí, como un pueblo. iEl monte es alegre (pausa), bueno yo me siento más contenta! A veces yo le digo a su papá de ellas: “¿Cuándo volvemos a hacer milpa como antes?”

5) La abundancia de presas de caza al principio de la ocupación.

\section{Los patrones materiales y espaciales y las estimaciones de población}

Los ranchos familiares que conformaban la ranchería se construían en terrenos altos para evitar las inundaciones y cuando mucho a un kilómetro de distancia

\footnotetext{
${ }^{9}$ Son dos aguadas en las que se desarrollaron rancherías y milperías a más de $25 \mathrm{~km}$ de distancia de Pich, Campeche.
} 
de la fuente de agua (aguada o pozo), con el fin de facilitar a las mujeres el acarrear agua para sus actividades domésticas. Esta fuente de agua debía ser de capacidad suficiente para abastecer a las familias todo el año. ${ }^{10}$ Estos ranchos incluían la casa (la sala-dormitorio y una cocina semiabierta), el huerto, los corrales para animales domésticos y el lavadero (Zetina, 2007: 83, 85-86, 92-93,109).

Las características constructivas del rancho familiar, sus edificaciones y sus lapsos de ocupación, dependieron del cálculo del tiempo que sus habitantes tardarían explotando: 1) las presas de caza; y 2) las tierras aptas para la agricultura (aunque no siempre se podía aprovechar todo el terreno al alcance de la ranchería, pues una parte podía ser inundable, o estar llena de espinas o zacatales difíciles de erradicar sin tractor o herbicidas). Estas proyecciones de lapsos de ocupación se materializaron en la elección de los materiales de construcción de las casas a manera de domicilios familiares, y los galerones (construcciones rústicas con cuatro postes que sostienen un techo de palma de huano y sin muros) como domicilios eventuales de algunos milperos cerca de sus campos de cultivo (Zetina, 2007: 132). En ranchos ocupados por más de cinco años, se seleccionaron buenas maderas para la estructura básica y se construyeron muros resistentes a los vientos y las lluvias junto con un buen techo de palma de huano. Por ejemplo, un informante de 92 años de edad mencionó que si hay monte bueno para quedarse unos seis años, la casa se debe construir bien (Zetina, 2007: 112).

Esto se traduce en un mayor número de vestigios sobre el paisaje: corrales de piedra, algunas piedras dispersas, basureros y una mayor variedad de plantas y árboles no locales, como el plátano y el mango, en lo que fueron los solares (Zetina, 2007: 38, 86). En un pueblo pequeño abandonado hace más de 15 años, todavía se encontraron los horcones y las estructuras de los techos in situ. Pero lo más notable fue que en relación con la construcción de las casas y de los galerones milperos, los informantes dijeron no utilizar piedras para las cimentaciones porque sabían que no necesitarían estas casas a largo plazo. ${ }^{11}$ Durante la época prehispánica, sin embargo, las construcciones con plataformas de piedra y muros de mampostería fueron comunes aún en los asentamientos dispersos, lo cual indica una permanencia relativamente larga. No es lógica tal inversión si la ocupación por largo tiempo no estaba asegurada.

Durante la Colonia no hubo continuidad en la construcción de plataformas y cimentación residenciales porque esto pudiera haber evidenciado la permanencia de la residencia familiar fuera de la "reducción de indios", lo que era castigado

\footnotetext{
${ }^{10}$ Por lo general, los requerimientos de agua para dos o tres milperos en el galerón durante la ocupación estacional (de unos días hasta tres semanas) sólo exigía una aguada pequeña que podía ser igualmente estacional (Zetina, 2007: 84-87). Algunas veces, cuando la aguada no estaba tan cerca, el agua se transportaba con carreta de mulas dentro de un contenedor metálico.

${ }^{11}$ Las observaciones directas en los restos de rancherías y en los pueblos abandonados mostraron escasas piedras en la superficie; esto indica que no se continuó con el modo de cimentar las casas en tiempos prehispánicos. Es un poco difícil pensar cómo una casa que se ocupó más de 10 años podía carecer de una cimentación de piedra al menos burda.
} 
(Farriss, 1984: 199-214, Bracamonte, 2001: 80). Por ello aprendieron a vivir con menor calidad y labor invertidas en sus viviendas fuera de los pueblos oficialmente registrados.

Referente a la distribución espacial de las rancherías, ésta era dispersa. Cada rancho familiar poseía aproximadamente una cuarta parte de hectárea o más, aunque a veces se ubicaban a más de $1 \mathrm{~km}$ unas de otras. Pero a pesar de estas distancias, el asentamiento era considerado una comunidad por sus pobladores pues se ayudaban mutuamente con sus tareas y si no eran parientes pasaban de la amistad al compadrazgo (Zetina, 2007: 83, 85-86, 92-93,109).

Respecto a las distancias entre las rancherías y la comunidad de Pich, éstas variaban entre 8 y $40 \mathrm{~km}$, pero es necesario tomar en cuenta que en el siglo xx ya se contaba con animales de tiro (caballo y mula), carretas y, más tarde, camiones de carga. El área inmediata a Pich se usaba para ganadería y para milpas de quienes residían permanentemente en el pueblo, generalmente milperos ancianos o enfermos sin muchas fuerzas para desplazarse. También existían ranchos privados con ganado y milpas.

Los terrenos agrícolas cercanos a la ranchería fueron los primeros en usarse, hasta que paulatinamente las milpas se iban alejando en un patrón de movilidad circular o en espiral hacia afuera. Cuando las milpas se alejaban más allá de 2 $\mathrm{km}$ entonces los milperos necesitaban construir un refugio temporal llamado localmente "galerón" o "champa" (bodega para implementos agrícolas construido sin muros y con materiales perecederos) para protegerlos de las lluvias (figura

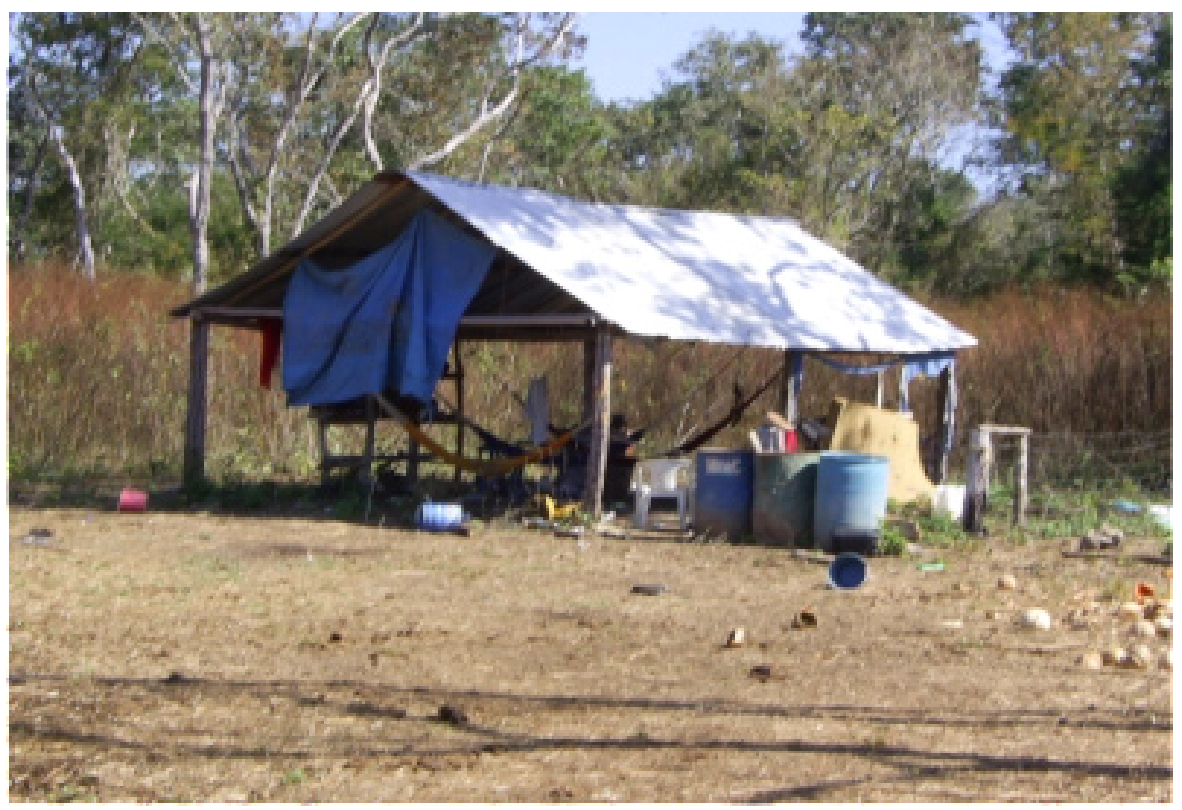

Figura 4. Galerón o champa milpera similar a las reportadas por los informantes (Zetina, 2007: 181) 
4). Normalmente, el galerón, no se construía a más de $2 \mathrm{~km}$ de la ranchería, es decir, la milpa no se establecía a más de $4 \mathrm{~km}$ de la ranchería donde se quedaron las mujeres y los niños pequeños. Con un límite de $2 \mathrm{~km}$ para desplazarse desde la ranchería hacia cada dirección, habría disponibles 4 por 4 km o $16 \mathrm{~km}^{2}$, equivalente a 1600 ha. Si cinco milperos tumbaron 2 ha y reusaron otras 2 ha, en el segundo año habría 4 ha usadas por cinco milperos, lo que resultaría en 20 ha por año. Así, usando un barbecho mínimo de 20 años, cinco agricultores requerirían solamente 400 ha de terrenos agrícolas antes de empezar un nuevo ciclo. De manera que, si todo el terreno era adecuado, una ranchería pudiera haber mantenido cuatro veces más gente (hasta 20 milperos y sus familias, esto es, 400 ha por $4=1600$ ha) por tiempo indefinido. Sin embargo, tenemos el dato de que se movieron en un promedio de 14 años, indicando una ventaja en hacer las milpas en la selva que tiene más de 20 años de crecimiento, si bien intervinieron también razones no agrícolas (Zetina, 2007: 135).

Se detectó que existía un ciclo doméstico de aproximadamente 35 años, el cual iniciaba cuando un joven padre maya empezaba a hacer su propia milpa y terminaba cuando ya ha perdido fuerza por la vejez y debe depender de sus hijos adultos para hacer la mayoría del trabajo. Por lo general, los milperos seguían trabajando su milpa hasta perder fuerza o morirse a una edad de 60 años o más.

Así que en una estimación paleodemográfica basada en un ciclo doméstico de aproximadamente 35 años, al mover la ranchería cada 14 años una familia nuclear tendría entre dos y tres casas en rancherías $(35 / 14=2.5)$, más otra en el pueblo. Esto es, entre tres y cuatro restos de residencias producto del mismo grupo familiar (Zetina, 2007: 139) en una generación. Estos datos resultan diferentes de las cinco unidades residenciales usadas y abandonadas en un ciclo doméstico (35 años), posibilidad presentada por Folan et al. con base en movimientos entre rancherías cada 8 o 10 años (2000: 10) y en la propuesta de Santley (1990: 333) de la erección de 10 a 15 estructuras por el mismo grupo familiar en un periodo de 200 años. Nos haría falta establecer para lograr cifras aún más exactas cuántos hijos en promedio tenía cada familia y si reusaban o no los cimientos de las casas en ranchería cuando la nueva generación volvió al mismo lugar a establecer su rancho. Decimos que 3 o 4 podría ser un número razonable; pero suponiendo que los lapsos de ocupación en época prehispánica pudieran ser más largos en comparación con los documentados en Pich, Campeche, podríamos reducir al mínimo el número de casas de una familia en una generación a 2 o 3. Y aún así serían significativos los resultados respecto a las cifras manejadas actualmente.

\section{Conclusiones}

Los resultados de esta investigación mostraron continuidad histórica en los patrones conductuales asociados a la agricultura de roza, tumba y quema, y al estilo de vida desarrollado en las rancherías de Pich, Campeche. Dichos patrones 
parecen ser compartidos y comunes a las Tierras Bajas Mayas (Campeche, Yucatán y parte de Guatemala), según la información documental reunida. Asimismo, sugieren ser resultado de una estrategia de adaptación ecológica que trata de asegurar un aprovisionamiento suficiente de comida, teniendo como base la agricultura, la caza, la horticultura y, en menor medida, la recolección.

El estilo de vida de las rancherías mayas, que subsistían explotando los recursos naturales locales, les permitía ser autosuficientes aunque el intercambio y el comercio existían en pequeña escala. La vida seminómada también resultaba provechosa para escapar del aburrimiento, de las epidemias humanas y animales, además de ser menos conflictiva y estresante al haber menos individuos a quienes organizar y con quienes competir por espacio y recursos. Las rancherías mayas resultaron ser verdaderas unidades socioeconómicas construidas sobre los lazos de parentesco consanguíneo o ritual (compadrazgo), y no ranchos familiares aislados en medio de su finca. Todo esto apoya la argumentación de Farriss (1984: 319) a favor de una tendencia cultural a la fragmentación o dispersión de grupos pequeños desde antes de la Colonia, al parecer debida a sus ventajas.

Como hemos revisado, la movilidad implica construcción y abandono de viviendas por una gran variedad de razones. Algunas de ellas, al ser agrícolas, son previsibles en lapsos regulares; otras, de naturaleza idiosincrásica o personal, son un poco más erráticas. Sin embargo, es mérito de esta investigación establecer las necesidades de terrenos regenerados durante más de veinte años de la milpa de roza, tumba y quema, y la búsqueda de presas de cacería como los principales promotores de una movilidad residencial mucho más frecuente de lo que se había pensado. Esta movilidad estaba regida por patrones cíclicos temporales y espaciales en varios niveles: la reocupación generacional (de 20 años o más entre una generación y otra), movimientos de una ranchería a otra cada 14 años en promedio, así como el movimiento de las milpas cada dos o tres años (figuras 2 y 3). Por otro lado, la bilocalidad entre la ranchería y el pueblo permitió conservar el acceso a los servicios civiles, religiosos y de mercado ofrecidos en los asentamientos más grandes

Con base en todos los datos etnográficos y documentales presentados, argumentamos que estos mismos comportamientos fueron comunes en las Tierras Bajas Mayas arraigados culturalmente de generación en generación. Esto significa que la mayoría de las familias de milperos mayas prehispánicos pudieron construir y abandonar, por lo menos, tres casas (dos en rancherías y una en el pueblo) en un ciclo doméstico de 35 años. Por ello, los resultados de las estimaciones paleodemográficas basadas en conteo de unidades residenciales en las Tierras Bajas Mayas deberían ser reducidas cuando se consideran los centros de población (pueblos y ciudades) y sus áreas de sustentación (donde se asientan las rancherías).

Tal reducción coincidiría con la capacidad de carga calculada para la milpa maya, aunque todavía no sabemos qué proporción del sustento de las ciudades mayas antiguas provino de esta forma de agricultura y cuál de los sistemas intensivos (Zetina, 2007). 
Respecto a la bilocalidad en contextos prehispánicos, de acuerdo con los datos de Abrams (1994) es posible que un milpero residiendo en ranchería estuviera una parte del año en los pueblos o ciudades cuando se requería la mano de obra masculina para la construcción de obras públicas o para realizar alguna actividad especializada. Esto se debe a que la suma de todos los días invertidos en tareas de la milpa es de alrededor de 72 al año, según los cálculos de Redfield y Villa Rojas (1962: 52-53).

Es importante hacer notar que la bilocalidad anual asociada a la atención de las milpas en etapas críticas (como la siembra o la cosecha), y que materialmente se expresaba en una casa en el pueblo y en el uso temporal de un galerón milpero como dormitorio, no afecta las estimaciones del tamaño de las poblaciones arqueológicas. Esto debido a que, metodológicamente, los conteos se hacen con estructuras identificadas como residenciales pues poseen espacios domésticos formales (plataformas, dormitorios, cocina, bodega, cisternas o chultunes o pozos, etc.) y materiales también de la esfera doméstica (cerámica utilitaria, metates, fogones, basureros, etc.), así como entierros que denotan permanencia. La bilocalidad permanente (una casa en el pueblo y otra en la ranchería) sí afecta los resultados.

De esta manera, queda claro que las unidades residenciales reportadas por los arqueólogos como sencillas en su construcción pero con los espacios formales de una casa de ninguna manera pueden ser considerados como estructuras usadas solamente por los milperos para sus tareas agrícolas y ser descartados del conteo. Sin embargo, el mayor problema sigue siendo que (como los ranchos familiares de Pich durante el siglo $\mathrm{xx}$ ) fueron construidos con materiales perecederos $\mathrm{y}$, por lo tanto, contienen menos elementos arquitectónicos susceptibles de reflejar una variación cronológica. En tiempos prehispánicos se invirtieron más labor y materiales en estas residencias, pero es difícil establecer la contemporaneidad dentro de un mismo periodo arqueológico (generalmente de entre 200 y 500 años). Con todo, es posible auxiliarse de los artefactos (como cerámica y lítica) y ecofactos (como polen, restos de plantas, etc.) asociados y de la estratigrafía para poder determinar dicha información.

Las cifras paleodemográficas estimadas para cada ciudad-estado maya probablemente son mucho más grandes de lo que debieron ser en la realidad, pues nunca se han aplicado correcciones matemáticas basadas en movilidad y bilocalidad (Zetina, 2007: 141). Así, se ha interpretado a cada unidad residencial sin aparente variación cronológica a partir de su arquitectura y sus materiales, como equivalente a una familia diferente. Los datos encontrados en las historias orales de Pich nos dan pistas que podrían frenar la desesperada búsqueda de cómo se pudo alimentar a una población considerada varias veces más grande que la actual (Zetina, 2007: 139). Estos mismos datos nos auxilian en la elaboración de un modelo donde una combinación de marcadores arqueológicos de distinta naturaleza podría mostrarnos la posibilidad de su existencia en tiempos precolombinos (figura 5). 


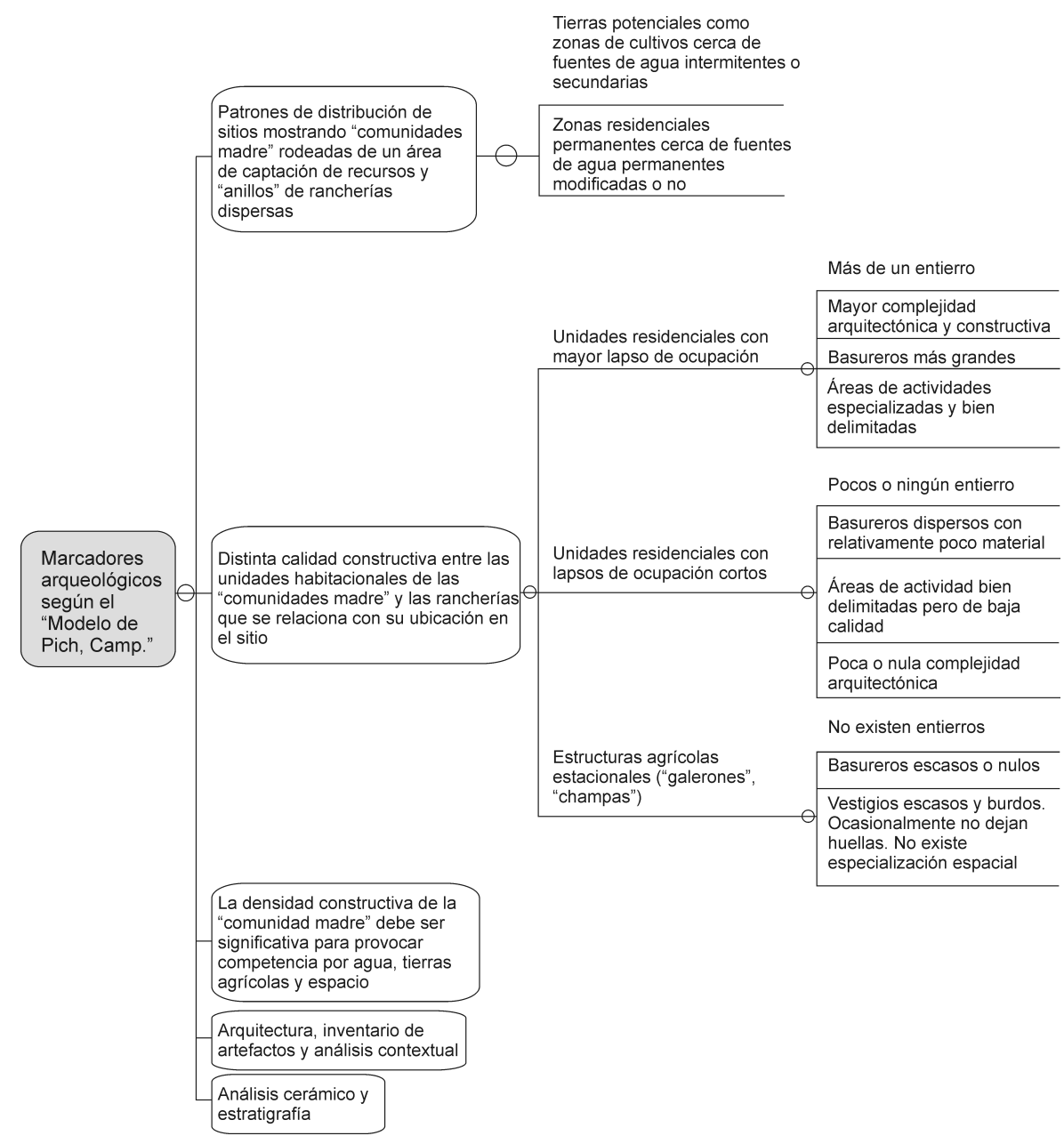

Figura 5. Diagrama mostrando los marcadores arqueológicos de la movilidad y la bilocalidad residencial

Es importante que los arqueólogos conozcan el funcionamiento y las necesidades de la milpa de roza, tumba y quema para poder interpretar de mejor manera los patrones espaciales y materiales de las comunidades o regiones que se estudian. Sin caer en un determinismo ecológico, podemos asegurar que el medio ambiente natural influye enormemente no sólo la distribución y la densidad constructiva sino también los materiales, el sistema constructivo de las unidades residenciales y los patrones de residencialidad, como ya hemos visto. 


\section{BIBLIOGRAFÍA}

Abrams, Elliot.

1994 How the Maya Built their World: Energetics and Ancient Architecture. Austin: University of Texas at Austin.

Becquelin, Pierre y Michelet Dominique

1994 "Demografía en la Zona Puuc: el recurso del Método", Latin American Antiquity, 5 (4): 289-311.

Bracamonte y Sosa, Pedro

2001 La conquista inconclusa de Yucatán. Los mayas de la montaña, 1560-1680. México: Centro de Investigaciones y Estudios Superiores en Antropología Social (Colección Peninsular).

Brown, Denisse Fay

1993 "Yucatec Maya Settling. Settlement and Spaciality", tesis para obtener el grado de Philosophy Doctor. Riverside: University of California Riverside, Department of Anthropology.

Conklin, Harold C.

1957 Hanunoo Agriculture: A Report on an Integral System of Shifting Cultivation. Roma: Food and Agriculture Organization of the United Nations.

Culbert, T. Patrick

1977 "Maya Development and Collapse: An Economic Perspective", Norman Hammond (ed.), Social Process in Maya Prehistory: Essays in Honor of Sir Eric Thompson. Londres: Academia Press, 509-530.

Culbert, T. Patrick y Don S. Rice (eds.)

1990 PreColumbian Populations History in the Maya Lowlands. Albuquerque [Estados Unidos]: University of New Mexico Press.

Dunning, Nicholas

1992 Lords of the Hills: Ancient Maya Settlements in the Puuc Region, Yucatan, México. Madison: Prehistory Press (Monographs in World Archaeology, 15).

Farriss, Nancy M.

1978 "Nucleation versus Dispersal: The Dynamics of Population Movement in Colonial Yucatan”, Hispanic American Historical Review, 58 (1978): 187-216.

1984 Maya Society under Colonial Rule: The Collective Enterprise of Survival. Princeton: Princeton University Press.

Faust, Betty B.

1988 "Cosmology and Changing Technologies of the Campeche Maya". Ph. D. dissertation. Syracuse: Syracuse University.

1998 Mexican Rural Development and the Plumed Serpent. Westport: Greenwood Publishers / Bergin and Garvey Press. 
Faust, Betty B.

2001 "Maya Environmental Successes and Failures in the Yucatan Peninsula", Environmental Science and Policy, 4 (4/5): 153-169.

2004 "The End of Innocence in a Modernizing Maya Community: 'There In No More Timber; Let's Sell the Land'”, Rights, Resources, Culture, and Conservation in the Land of the Maya, Betty B. Faust, E. N. Anderson y John G. Frazier (eds.). Westport [Estados Unidos] / Londres: Praeger.

Faust, Betty B. y Richard Bilsborrow

2000 "Maya culture, population, and the environment on the Yucatán Peninsula", Population, Development and Environment on the Yucatán Peninsula: from Ancient Maya to 2030, Wolfgang Lutz, Leonel Prieto y Warren Sanderson (eds.). Laxenburg [Austria]: International Institute for Applied Systems Analysis, 73-107.

Fedick, Scott L. (ed.)

1996 The Managed Mosaic. Ancient Maya Agriculture and Resource Use. Salt Lake City: University of Utah Press.

Flannery, Kent V. (ed.)

1982 Maya Subsistence. Studies in Memory of Dennis Puleston. Nueva York: New York Academic Press.

Folan, William J., Betty B. Faust, Wolfgang Lutz y Joel D. Gunn

2000 "Social and environmental factors in the classic maya collapse", Population, Development and Environment on the Yucatán Peninsula: from Ancient Maya to 2030, Wolfgang Lutz, Leonel Prieto y William Sanderson (eds.). Laxenburg [Austria]: International Institute for Applied Systems Analysis, 2-32.

Folan, William, Ellen R. Kintz y Loraine A. Fletcher

1983 Coba: A Classic Maya Metropolis (Studies in Archaeology). Nueva York: Academic Press.

Ford, Anabel

1990 "Maya settlement in the Belize River area: variations in residence patterns of Central Maya Lowlands", Precolumbian Populations History in the Maya Lowlands, Patrick Culbert y Don S. Rice (eds.). Albuquerque: University of New Mexico Press, 167-181.

Gendrop, Paul

1997 Diccionario de arquitectura prehispánica. México: Trillas.

1983 Los estilos Río Bec, Chenes y Puuc en la arquitectura maya. México: Universidad Nacional Autónoma de México, División de Estudios de Posgrado de la Facultad de Arquitectura.

Gómez-Pompa, Arturo, Michael F. Allen, Scott. L. Fedick y Juan. J. Jiménez-Osornio (eds.) 2003 The Lowland Maya Area: Three Millennia at the Human-Wildland interface. Binghampton [Estados Unidos]: Food Products Press.

Gutiérrez Martínez, José A.

1993 "Agricultura de roza y dinámica demográfica en una comunidad maya", Etnoecológica, 1 (2): 35-47. 
Harris, Daniel R.

1972 "Swidden Systems and Settlement", Man, Settlement and Urbanism, Peter J. Ucko, Ruth Tringham y G. W. Dimbleby (eds.). Cambridge [Estados Unidos]: Schenkman Publishing Company, 245-262.

Harrison, Peter D.

1977 "The Rise of the Bajos and the Fall of the Maya", Norman Hammond (ed.), Social Process in Maya Prehistory: Studies in Honor of Sir Eric Thompson, Londres: London Academic Press, 469-508.

1993 "Aspects of Water Management in the Southern Maya Lowlands", Economic Aspects of Water Management in the Prehispanic New World. Research in Ecomic Anthropology, Supplement 7, Vernon L. Scarborough y Barry L. Issac (eds.). Greenwich [Estados Unidos]: Jai Press.

Harrison, Peter D. y Billie L. Turner II

1978 Pre-Hispanic Maya Agriculture. Albuquerque: University of New México.

Haviland, William A.

1969 "A New Population Estimate for Tikal, Guatemala", American Antiquity, 34: 429-433.

Kintz, E. y L. Fletcher

1983 "A reconstruction of the prehistoric population at Coba", Coba. A Classic Maya Metropolis. William J. Folan, Ellen R. Kintz y Lorrain A. Fletcher (eds.). Nueva York: Academic Press: 191-210.

Kunen, Julie L.

2004 Ancient Maya Life in the Far West Bajo: Social and Environmental Change in the Wetlands of Belize. Tucscon: University of Arizona Press (Anthropological Papers of the University of Arizona, 69).

Landa, fray Diego de

1997 Relación de las cosas de Yucatán. México: Fondo de Cultura Económica.

[1566]

Matheny, Ray T., Deanne L. Gurr, Donald W. Forsyth y Forrest R. Hauck

1983 Investigations at Edzná Campeche, México. Vol. 1, part 1: The Hydraulic System. Provo [Estados Unidos]: Brigham Young University (Papers of the New World Achaeological Foundation, 46).

Meggers, Betty J. y Eurico Miller

2006 "Evidencia arqueológica para el comportamiento social y habitacional en la Amazonía prehistórica”, Pueblos y paisajes antiguos de la selva amazónica, Gaspar Morcote, Santiago Mora y Carlos E. Franky (eds.). Bogotá / Washington, D.C.: Universidad Nacional de Colombia / Tarazacum, 325-348.

Netting, Robert M.

1994 "Subsistencia maya: mitologías, analogías y posibilidades", Los orígenes de la civilización maya, 2a ed. México: Fondo de Cultuta Económica, 327-365. 
Pérez Toro, Augusto

1942 La milpa. Mérida: Gobierno de Yucatán.

Pohl, Mary D.

1990 Ancient Maya Wetland Agricultura: Excavations on Albion Island, Northern Belice. Boulder [Estados Unidos]: Westview Press.

Pool Novelo, Luis

1997 "Intensificación de la agricultura tradicional y cambios en el uso del suelo”, Los Altos de Chiapas: agricultura y crisis rural, Manuel Parra Vázquez y Blanca M. Díaz Hernández (eds.). Chetumal: El Colegio de la Frontera Sur, $1-22$.

Redfield, Robert y Alfonso Villa Rojas

1962 Chan Kom: A Maya Village. Chicago: University of Chicago Press.

[1934]

Sanders, William T. y Barbara J. Price

1968 Mesoamerica: The Evolution of a Civilization. Nueva York: Random House.

Santley, Robert

1990 "Demographic in the Maya Lowlands", PreColumbian Population History in the Maya Lowland, Patrick T. Culbert y Don S. Rice (eds.). Albuquerque: University of New Mexico Press, 325-343.

Scarborough, Vernon

1983 "Raised Field Detection at Cerros, Northern Belize", Drained Field Agriculture in Central and South America. Proceedings of the $44^{\text {th }}$ International Congress of Americanists, Janice P. Darch (ed.). Oxford: British Archeological Research International Series (189), 123-36.

Siemens, Alfred H.

1978 "Karst and the Pre-Hispanic Maya in the Southern Lowlands", Pre-Hispanic Maya agriculture, Peter D. Harrison y B. L. Turner (eds.). Albuquerque: University of New México Press, 117-143.

Siemens, Alfred H. y D. Puleston

1972 "Ridged Fields and Associated Features in Southern Campeche New Perspectives on the Lowland Maya”, American Antiquity, 37 (2): 228-239.

Tax, Sol

1937 "The Municipios of the Midwestern Highlands of Guatemala", American Anthropologist, (39): 423-444.

Terán, Silvia y Christian Rasmussen

$1994 \quad$ La milpa de los mayas. La agricultura de los mayas prehispánicos y actuales en el Noreste de Yucatán. Mérida: Danida / Gobierno del Estado de Yucatán.

Tourtellot, Gair

1990 "Population Estimates for Preclassic and Classic Seibal, Peten”, PreColumbian 
Population History in the Maya Lowlands, T. Patrick Culbert y Don S. Rice (eds.). Albuquerque: University of New Mexico Press, 83-102.

Tourtellot, Gair, Jeremy Sabloff y Michael Smyth

1990 "Room Counts and Population Estimation for Terminal Classic Sayil in the Puuc Region, Yucatán, México", PreColumbian Population History in the Maya Lowlands, T. Patrick Culbert y Don Rice (eds.), Albuquerque: University of New Mexico Press, 245-262.

Turner, Billie. L. II

1979 "Prehispanic Terracing in the Central Maya Lowlands: Problems of Agricultural Intensification”, Mesoamerican Archaeology and Ethnology, Norman Hammond y Gordon R. Wiley (eds.). Austin: University of Texas Press, 103-113.

1983 Once Beneath the Forest: Prehistoric Terracing in the Rio Bec Region of the Maya Lowlands. Boulder: Westview Press.

Webster, David L. y Anne C. Freter

1990 "The Demography of Late Classic Copan”, PreColumbian Populations History in the Maya Lowlands, T. Patrick Culbert y Don S. Rice (eds.). Albuquerque: University of New Mexico Press, 37-61.

Webster, David L., Anne C. Freter y Nancy Gonlin

2000 Copán: The Rise and Fall of An Ancient Maya Kingdom. Fort Worth [Estados Unidos]: Hartcourt College Publishers.

Zetina Gutiérrez, María de Guadalupe

2003 "Aproximaciones a los patrones de asentamiento y demografia en los sectores intersitios del distrito de Santa Elena, Región Puuc, Yucatán; México", tesis de licenciatura en Ciencias Antropológicas con especialidad en Arqueología. Mérida: Universidad Autónoma de Yucatán, Facultad de Ciencias Antropológicas.

2004 "Los patrones de asentamiento y sus métodos de campo en las tierras bajas del norte: perspectiva arqueo-ecológica”, ponencia presentada en el XIV Encuentro Internacional "Los Investigadores de la Cultura Maya" organizado por la Universidad Autónoma de Campeche, Campeche.

2007 "Ecología Humana de las rancherías de Pich, Campeche: Un estudio diacrónico", tesis de maestría en Ciencias. Mérida: Centro de Investigación y de Estudios Avanzados del Instituto Politécnico Nacional, Departamento de Ecología Humana.

Zetina Gutiérrez, María de Guadalupe y Betty Faust

2006 "The shift from nomadic agriculture to tractors in Pich, Campeche", ponencia presentada en el 105th American Anthropological Association Annual Meeting, noviembre de 2006, San José, California.

2007 "How many houses per family? Oral histories of nomadic agriculture in Campeche", ponencia presentada en el VII Congreso Internacional de Mayistas, organizada por la Universidad Nacional Autónoma de México, julio de 2007, Mérida. 